\title{
Comparative study on the performance of textural image features for active contour segmentation
}

\author{
MORARU Luminita ${ }^{*} \&$ MOLDOVANU Simona \\ Dunarea de Jos University of Galati, Galati RO-800008, Romania
}

Received March 8, 2012; accepted June 8, 2012

\begin{abstract}
We present a computerized method for the semi-automatic detection of contours in ultrasound images. The novelty of our study is the introduction of a fast and efficient image function relating to parametric active contour models. This new function is a combination of the gray-level information and first-order statistical features, called standard deviation parameters. In a comprehensive study, the developed algorithm and the efficiency of segmentation were first tested for synthetic images. Tests were also performed on breast and liver ultrasound images. The proposed method was compared with the watershed approach to show its efficiency. The performance of the segmentation was estimated using the area error rate. Using the standard deviation textural feature and a $5 \times 5 \mathrm{kernel}$, our curve evolution was able to produce results close to the minimal area error rate (namely $8.88 \%$ for breast images and $10.82 \%$ for liver images). The image resolution was evaluated using the contrast-to-gradient method. The experiments showed promising segmentation results.
\end{abstract}

active contour model, image feature, area error rate

Citation: Moraru L, Moldovanu S. Comparative study on the performance of textural image features for active contour segmentation. Sci China Life Sci, 2012, 55: 637-644, doi: 10.1007/s11427-012-4344-5

The segmentation of ultrasound images is necessary in clinical applications of medical imaging, but automatic techniques are still far from being fully developed. Segmentation allows us to distinguish objects from the background, and the most popular methods are pixel-based, region-based, edge-based and model-based [1].

Ultrasound image segmentation is used to obtain qualitative and quantitative information about objects from an image. In clinical practice, manual techniques to segment ultrasound images are often used, but they are laborious and tiring. The repetitive and monotonous delineation of tissue formation contours and distinguishing of contour variations by radiologists give rise to an increase in delineating faults. More sophisticated techniques are demanded. Various semiautomatic segmentation techniques and their clinical applicability have been reviewed [2,3]. Active contour meth-

*Corresponding author (email: luminita.moraru@ugal.ro) ods for image segmentation allow a contour to accommodate to the variability of the biological structures. Initially, this method establishes the contour and allows it to move to the boundary of the object using an energy function. Two types of active contour models, namely parametric active contour models (ACMs) and geometric active contours models, are typical semiautomatic segmentation techniques [4]. Active contours were introduced by Kass et al. [5] and developed by Terzopolus [6] to allow the segmentation of objects using dynamic snakes or curves. Lefebvre et al. [7] used an ACM to automatically determine the boundary of the calcaneus from ultrasound images. Their method correctly detected the boundary in the great majority of cases. A computer-aided method using a variable background active contour model was implemented by Maroulis et al. [8] to delineate thyroid nodules in thyroid ultrasound images.

The snake approach has been successfully applied to liver ultrasound images to detect hepatic tumors [9] or to ex- 
tract the shapes of gallbladders in the early diagnosis of gallbladder lesions [10]. A snake model that automatically detects the intimal and adventitial layers of the common carotid artery from ultrasound imagery was proposed by Cheng et al. [11], and developed by Mao et al. [12] and Loizou et al. [13]. An automatic initial contour-finding method for boundary detection in ultrasound images was established by Chen et al. [14] and Huang and Chen [15]. To quantify the cardiac function, a method based on an extension of the ACMs was designed and the left-ventricular epicardial and endocardial borders were traced into echocardiographic images [16,17]. A combination of image processing techniques has been used for more effective segmentation. For example, Mudabhushi and Metaxas [18] used low-level image filtering, speckle reduction, probabilistic classification of image pixels based on intensity and texture as well as deformable models to segment breast lesions from ultrasound images.

ACMs were also used to locate the object boundaries in various applications of medical image processes such as segmentation of different abnormalities in the human liver [9], heart [16], calcaneus [7] and breast [19]. As a first step, the active contours are manually initialized near an organ boundary, and the process automatically deforms the contour toward the boundary. The main drawbacks are the need for accurate initialization, correct tuning of the deformation parameters, and iteration to a local minima. The mentioned research papers deal with active contours and present various methods to improve initialization, speed, and robustness.

In this paper, the ACM and standard deviation texture feature are adapted for ultrasound images of breast cysts and the polycystic liver, and their efficiency and accuracy are tested on synthetic images. Our original contribution consists of replacing the image function of the ACM with the image features described below. The specific goal is to determine the best result of employing the method to promote robust segmentation of actual B-mode ultrasound data. Synthetic ultrasound images are used to test the algorithm since the true object boundary is known. This approach allows us to compare our results with the boundaries of interest in images hand-drawn by radiologists. Following this, the accuracy of the method is evaluated.

For comparison, the watershed algorithm for image segmentation is also used. Watershed and snake methods are extensively used in the segmentation of ultrasound images, particularly in locating object boundaries. Breast and liver ultrasound images that have frequent boundary discontinuities are often problematic in the extraction of accurate contours. In the first effort to overcome such problems, the watershed method has been successfully applied. It has the ability to create closed boundaries and it has a fast implementation algorithm; however, the watershed scheme has a major problem of over-segmentation [20]. Huang and Chen [21] employed initial over-segmentation using a watershed algo- rithm, and then used an ACM to refine the segmentation results. Sharma et al. [22] demonstrated that the ACM outperforms the watershed method and extracts a more accurate area than the watershed method. Moreover, Zhao and Zhuang [23] proposed a hybrid method using both watershed and snake methods in boundary detection. Our choice of the watershed algorithm is justified because the algorithm is able to produce a complete division of the image. Additionally, the contrast-to-gradient (CG) method for evaluating image resolution [24] and the execution time are used as qualitative assessment tools.

This paper is organized as follows. Section 1 presents the methods and mathematical description of the proposed ACMs. Experimental results are given in Section 2. Results and discussion are presented in Section 3. Finally, the paper is concluded in Section 4.

\section{Materials and methods}

The proposed method basically comprises two steps: (i) a pre-processing phase in which the original image is mapped using moving standard-deviation kernels to obtain a new image feature $I_{\mathrm{SD}}$ and (ii) image segmentation using an ACM. The desired contour is accordingly identified, using the ACM and the new image feature. These two steps are applied to synthetic and in vivo images in this work.

Figure 1 depicts the concept of contour movements used to fix an image as edges or a border. This concept has been adapted to our goals. The darker sample represents the analyzed object and the black points are the initial nodes of the snake. The point $S(1)$ marks the start point of the curve and the point $S(n)$ marks the end point.

The ACM or snake is a parametric curve defined in an image domain as $S(u)=I[x(u), y(u)], u \in[0,1]$. This curve represents the position of a snake that deforms the shape and moves within the spatial domain of an image to minimize the energy function $[4,5]$ :

$$
E=\int_{0}^{1} E_{\text {snake }}(S(u)) \mathrm{d} u,
$$

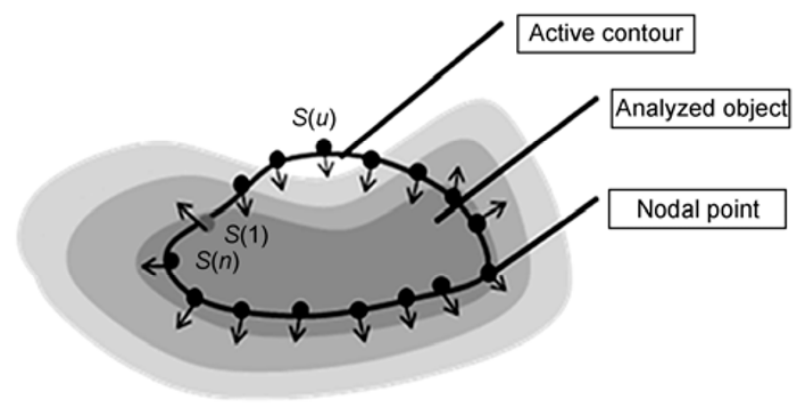

Figure 1 Illustration of the ACM method. The point $S(1)$ marks the start point of the curve and the point $S(n)$ marks the end point. 
where $E_{\text {snake }}$ is the snake's energy at a certain point [25]. This energy function is used to move the snake across an image.

Eq. (2) describes the total energy of snakes:

$$
E=\int_{0}^{1}\left[E_{\text {int }}(S(u))+E_{\text {img }}(S(u))+E_{\text {con }}(S(u))\right] \mathrm{d} u,
$$

where $E_{\text {int }}(S(u))$ is the internal energy of the spline and $E_{\text {img }}(S(u))$ gives rise to the image energy, and $E_{\text {con }}(S(u))$ gives rise to the external constraint forces [5]. The internal energy presented by eq. (3) is composed of first- and second-order derivatives, controlled by the weighting parameters $\alpha$ (i.e., the elasticity of the snake or the ability of the snake to act as a membrane) and $\beta$ (i.e., the rigidity of the snake or the ability of the snake to act as a thin plate):

$$
E_{\text {int }}=\frac{\alpha}{2} \underbrace{\left.\frac{\partial}{\partial u} S(u)\right|^{2}}_{\text {Elastic energy }}+\frac{\beta}{2} \underbrace{\left|\frac{\partial^{2}}{\partial u^{2}} S(u)\right|^{2}}_{\text {Bending energy }} .
$$

Image energy $E_{\text {img }}$ is used to drive the contour towards the desired image features, such as boundaries. In the well-established ACM [26], this energy function is estimated taking into account the results of the edge detection and it is calculated following the mathematical formalism presented below.

At $(x, y)$ in a gray-level image $I(x, y)$, finding the edges can be described using the energy functions [27]:

$$
\begin{gathered}
E_{\text {edge }}=-|\nabla I(x, y)|^{2}, \\
E_{\text {img }}=-\left|\nabla G_{\sigma}(x, y) * I(x, y)\right|^{2},
\end{gathered}
$$

where $G_{\sigma}(x, y)$ is a two-dimensional Gaussian kernel with standard deviation $\sigma . \nabla$ and $*$ indicate the gradient and convolution operator, respectively. In eq. (5), to reduce the noise effect on the image, Gaussian filtering is applied [28].

If salient image features (namely strong edges) are presented, the contour will be easily driven towards the object boundaries. The problem becomes more complicated in the absence of strong edges, when the model cannot correctly detect objects and it tends to collapse. During its movement, the snake encounters edge fragments caused by noise that may keep it from reaching the contour of the object. The limits and drawbacks of the snake are associated with initialization and poor convergence in noisy images.

It is well known that $E_{\text {img }}$ depends solely on the values of image intensity along the path of the spline. An important step in employing the snake model is providing an appropriate term for the image energy, because the snake will try to position itself in areas of low energy. In view of controlling over-segmentation and overcoming these limitations, we replaced the original image $I(x, y)$ with the function in eq. (6) that provides a smooth image so that the snake will be more attracted towards contours with large image gradients. In our approach, an image feature representing a new image is reconstructed according to the content of the original image, even if the value of each pixel is replaced by the local standard deviation values computed in $k \times k(k=3,5,7)$ masks. This new image feature $I_{\mathrm{SD}}$ function integrates the image information and pixel relation:

$$
I_{\mathrm{SD}}=\frac{1}{n^{2}-1} \sqrt{\sum_{(i, j) \in V_{n}(x, y)}[I(i, j)-M(x, y)]^{2}},
$$

where $M(x, y)$ denotes the average gray-level values in the neighborhood $V_{n}(x, y)[7,29]$ :

$$
M(x, y)=\frac{1}{n^{2}} \sum_{(i, j) \in V_{n}(x, y)} I(i, j) .
$$

This function can be interactively controlled by choosing an appropriate initial contour. Its main advantage is its sensitivity to the image scale due to the smoothing operation on the image energy function.

In this new image, the value of each pixel is equal to the local standard deviation of the gray-level values calculated from the original image using the chosen kernels. We avoided using larger kernels because they usually produce an image feature without strong edges and therefore such a model would be unable to correctly detect objects and would tend to collapse. If the kernel is small in size, more iteration will be needed to scan the object.

To surpass the discrepancy of image sizes, each image feature $I_{\mathrm{SD}}$ was bordered with a frame having the same dimensions as the selected mask.

The iterative algorithm implemented to extract the image features based on formulas (6) and (7) is as follows.

Input: Original image: $I, n$ number of kernels

$$
\begin{aligned}
& {[m, p]=\operatorname{size}(I)} \\
& {[w, h]=\operatorname{size}(\text { kernel })} \\
& \text { for } i \leftarrow 1 \text { to } n \text { do } \\
& \text { for } i \leftarrow 1 \text { to: } m-w \text { do } \\
& \text { for } j \leftarrow 1 \text { to } p-h \text { do } \\
& \text { kernel } \leftarrow \mathrm{SD}(I(i: i+w, j ; j+h)) \\
& I_{\mathrm{SD}} \leftarrow \text { kernel } \\
& w \leftarrow w+2 \\
& h \leftarrow h+2 \\
& \text { end } \\
& \text { end }
\end{aligned}
$$

Output: $I_{\mathrm{SD}}$

Synthetic ultrasound images (Figure 2) were used to test our solution because the simulated object boundary is known for these images. An ultrasound image of a circular object in a homogeneous background was created using Macromedia Fireworks 8 software [30]. The goal was to determine the compatibility between the proposed image 

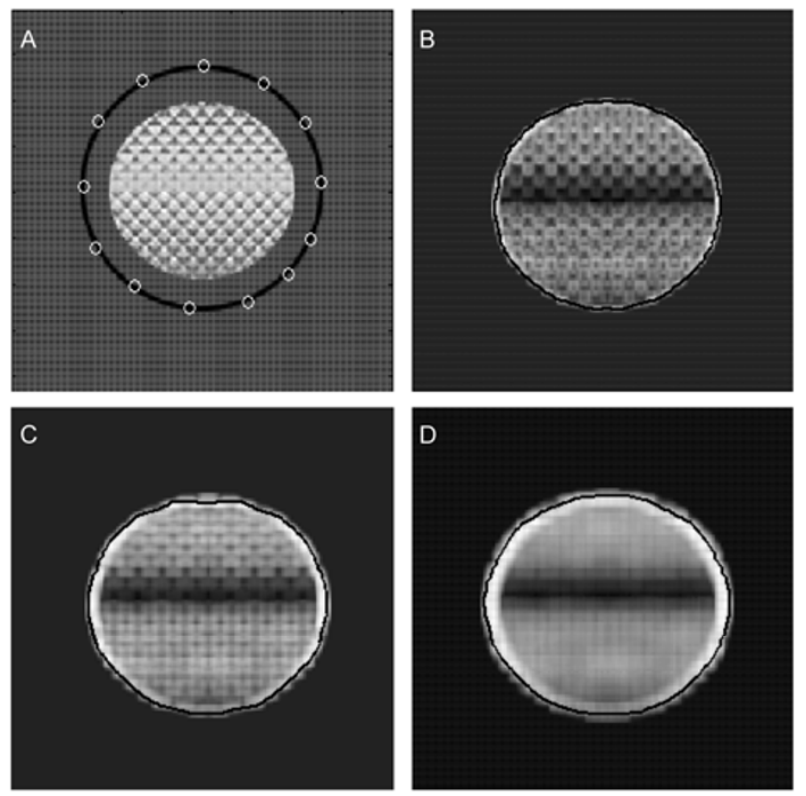

Figure 2 An example of synthetic image, image features and the final state of the snake. A, Initial contour and original synthetic image of disk shape. B, The image feature $I_{\mathrm{SD}}$ and its overlap with the ACM for a $3 \times 3$ kernel. C, The image feature $I_{\mathrm{SD}}$ and its overlap with the ACM for a $5 \times 5$ kernel. D, The image feature $I_{\mathrm{SD}}$ and its overlap with the ACM for a $7 \times 7$ kernel. The images show the snake in black.

features $I_{\mathrm{SD}}$ and the ACM.

When the algorithm was implemented in Matlab software, the elasticity parameter $\alpha=0.4$ and rigidity parameter $\beta=$ 0.2 were kept unchanged for all snakes in all tested images, but the iteration number of the algorithm and the circle radius were guided by the size of the cysts.

A well-established and accepted criterion for evaluating the performance of segmentation algorithms does not yet exist. The area error rate (AER) was used for contour validation and quantitative assessment of the performance of the proposed algorithm for both synthetic and in vivo images [10]. The AER allows estimation of the difference (in percent) between the occupied areas of images. The math- ematical details were presented by Ciecholewski [10]. Here we briefly review the notations: $L v_{\text {accon }}$ is the fragment of the image obtained using the ACM; $L v_{\text {manual }}$ is the image fragment extracted manually; $U R=L v_{\text {accon }} \cup L v_{\text {manual }}$ and $I R=L v_{\text {accon }} \cap L v_{\text {manual }}$. The AER value is defined as

$$
\mathrm{AER}=\frac{a_{\mathrm{UR}}-a_{\mathrm{IR}}}{a_{\mathrm{MSR}}} \times 100 \%,
$$

where $a_{\mathrm{UR}}$ is the number of pixels in the area UR, $a_{\mathrm{IR}}$ is the number of pixels in the area IR, and $a_{\mathrm{MSR}}$ is the number of pixels in the manually extracted area denoted MSR. The MSR is the areas of cysts manually obtained by specialist physicians. In our approach, area variation exceeding $12 \%$ indicates over-segmentation or under-segmentation.

Here, the contrast-to-gradient (CG) method proposed by Ishitani [24] was used to estimate the image resolution. The CG resolution is defined as the weighted harmonic mean of the local resolution. The CG method is a powerful tool for the robust evaluation of image resolution.

The hardware of the experimental environment was an Intel (R) Core (TM) 2 Duo CPU T5900, 2.20 with 3-Gb RAM, operating Toolbox Processing Image within MATLAB R2009a. One synthetic image, 16 clinical breast cyst images and 10 polycystic liver images were used to test the proposed algorithm, and 81 contours were finally obtained. The images were acquired using a commercial imaging scanning system SLE-401 (manufactured by Medelkom Ltd.) and a linear probe with a frequency of 6.5-9 MHz. Each pixel in the acquired image had resolution of 8 bits. Two experienced radiologists manually delineated all tumor contours.

\section{Results}

Examples of breast and liver cysts in ultrasound images are shown in Figure 3A and D respectively. The measurements of the performance and efficiency of our common frame-
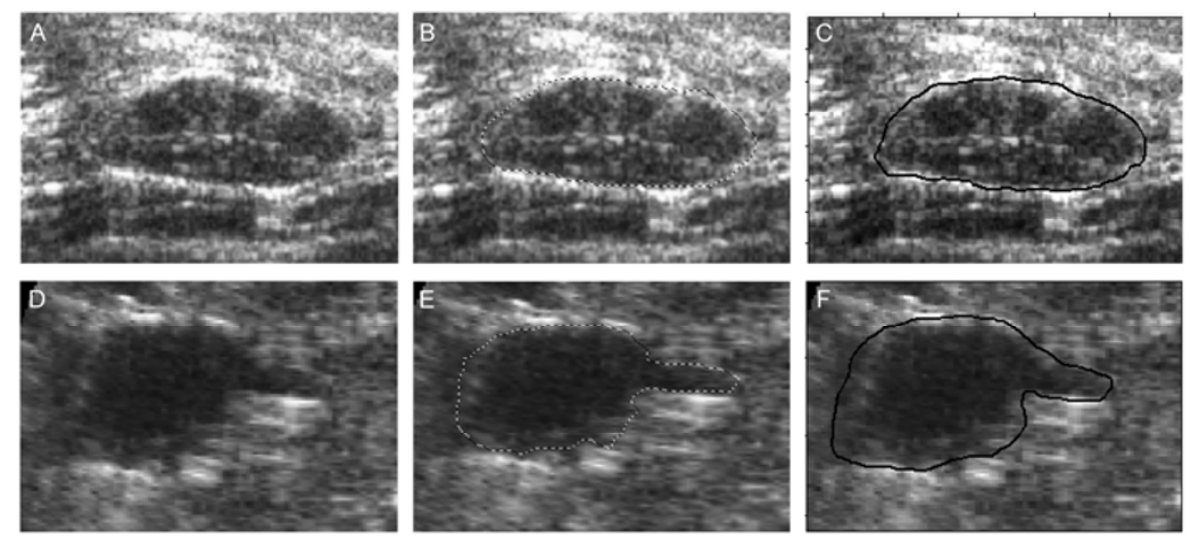

Figure 3 Examples of breast and liver echography images, image features and the final state of the snake. A, An example of a breast cyst. B, Cyst boundary (marked by a dashed line) delineated by a radiologist. C, The active contours or snakes in black and their overlap in the original breast image. D, An example of a liver cyst. E, Liver boundary (marked by a dashed line) delineated by a radiologist. F, The active contours or snakes in black and their overlap in the original liver image. 
work are presented in Tables 1 and 2, and examples of the results are shown in Figures 4 and 5.

A synthetic image in the shape of a disk was drawn and the snake was initialized as a circle in this synthetic image (Figure 2A). The synthetic image had a radius of 40 pixels and the snake had a radius of 50 pixels. Here, the nodal points that represent the initial position of the snake were fixed. The next step obtained the image features $I_{\mathrm{SD}}$ of the synthetic image using the standard deviation of the gray-level value calculated for kernel sizes of $3 \times 3,5 \times 5$ and $7 \times 7$. This step was iteratively repeated for each image feature. The convergence of the ACM onto image features is shown in Figure 2B-D.

For in vivo ultrasound images, the compatibility between the first-order feature energy function and the ACM was

Table 1 AERs of breast cyst ultrasound images segmented using the proposed method

\begin{tabular}{ccccc}
\hline & \multicolumn{4}{c}{ AER $(\%)$ in average } \\
\cline { 2 - 5 } Images & $\begin{array}{c}\text { Original } \\
\text { image }\end{array}$ & $\begin{array}{c}I_{\mathrm{SD}} \\
3 \times 3 \text { kernel } \\
\text { size }\end{array}$ & $\begin{array}{c}I_{\mathrm{SD}} \\
5 \times 5 \text { kernel } \\
\text { size }\end{array}$ & $\begin{array}{c}I_{\mathrm{SD}} \\
7 \times 7 \text { kernel } \\
\text { size }\end{array}$ \\
\hline Synthetic images & 5.64 & 12.50 & 4.79 & 18.40 \\
Breast cyst images & & & & \\
1 & 13.38 & 25.00 & 7.61 & 16.05 \\
2 & 12.50 & 26.85 & 12.76 & 12.94 \\
3 & 13.21 & 25.98 & 2.53 & 15.75 \\
4 & 14.49 & 20.85 & 11.07 & 10.13 \\
5 & 16.63 & 24.02 & 8.62 & 13.02 \\
6 & 19.24 & 29.83 & 7.04 & 11.75 \\
7 & 20.20 & 25.26 & 7.03 & 16.34 \\
8 & 14.43 & 23.84 & 9.49 & 13.44 \\
9 & 17.86 & 29.62 & 6.72 & 13.89 \\
10 & 15.43 & 24.48 & 9.09 & 14.09 \\
11 & 14.51 & 23.57 & 8.64 & 17.48 \\
12 & 11.08 & 27.16 & 12.14 & 9.05 \\
13 & 10.10 & 26.58 & 19.89 & 17.12 \\
14 & 17.50 & 26.75 & 10.73 & 15.32 \\
15 & 14.17 & 23.55 & 11.05 & 7.03 \\
16 & 13.76 & 29.07 & 6.70 & 15.97 \\
Average values & 14.90 & 25.77 & 8.88 & 13.71 \\
\hline
\end{tabular}

Table 2 AERs of liver ultrasound images segmented using the proposed method

\begin{tabular}{ccccc}
\hline & \multicolumn{4}{c}{ AER $(\%)$ in average } \\
\cline { 2 - 5 } Images & $\begin{array}{c}\text { Original } \\
\text { image }\end{array}$ & $\begin{array}{c}I_{\mathrm{SD}} \\
3 \times 3 \text { kernel } \\
\text { size }\end{array}$ & $\begin{array}{c}I_{\mathrm{SD}} \\
5 \times 5 \text { kernel } \\
\text { size }\end{array}$ & $\begin{array}{c}I_{\mathrm{SD}} \\
7 \times 7 \text { kernel } \\
\text { size }\end{array}$ \\
\hline 1 & 12.6 & 18.65 & 12.32 & 15.36 \\
2 & 17.9 & 21.36 & 14.04 & 17.98 \\
3 & 22.14 & 21.23 & 7.58 & 10.85 \\
4 & 11.09 & 22.02 & 12.23 & 11.47 \\
5 & 17.23 & 20.58 & 14.87 & 13.58 \\
6 & 19.47 & 19.21 & 9.26 & 16.51 \\
7 & 23.25 & 18.95 & 8.29 & 13.84 \\
8 & 21.95 & 16.28 & 7.59 & 11.09 \\
9 & 16.25 & 23.78 & 13.56 & 12.43 \\
10 & 20.51 & 21.89 & 9.32 & 14.09 \\
Average values & 16.55 & 20.27 & 10.82 & 14.72 \\
\hline
\end{tabular}
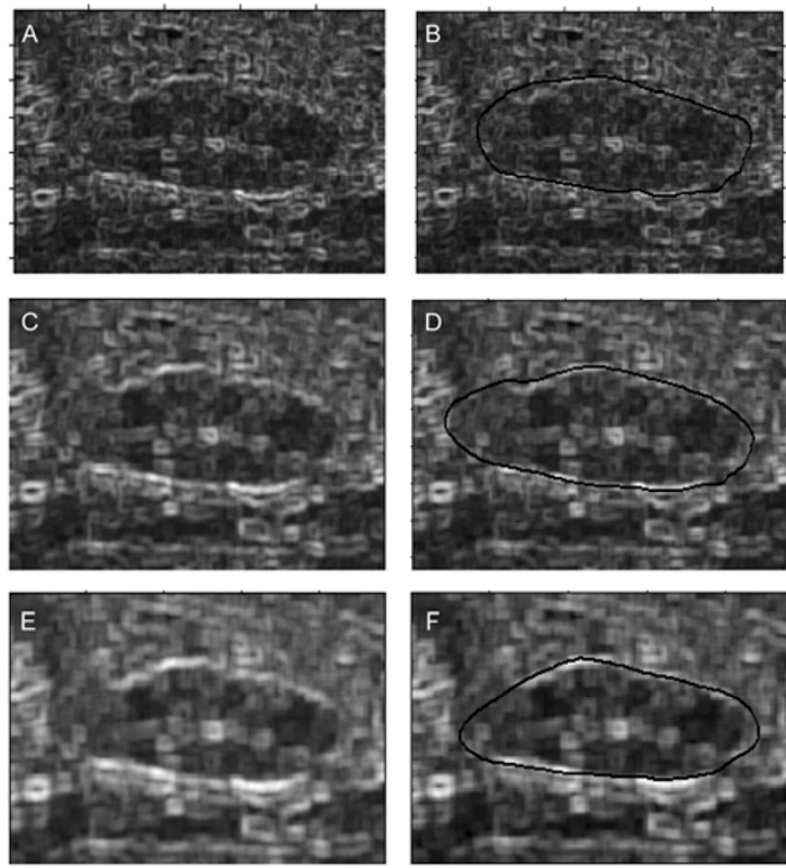

Figure 4 Image feature corresponding to the image presented in Figure $3 \mathrm{~A}$. A, C and E, The image features $I_{\mathrm{SD}}$ for $3 \times 3,5 \times 5$ and $7 \times 7$ kernels extracted from the breast cyst ultrasound image. B, D, and F, The active contours or snakes (in black) of boundaries derived using $3 \times 3,5 \times 5$ and $7 \times 7$ kernels. A large kernel (such as a $7 \times 7$ kernel) will produce blurred boundaries. As a result, the capture range is increased and the potential function becomes less sharp and the boundary localization will become less accurate and distinct.
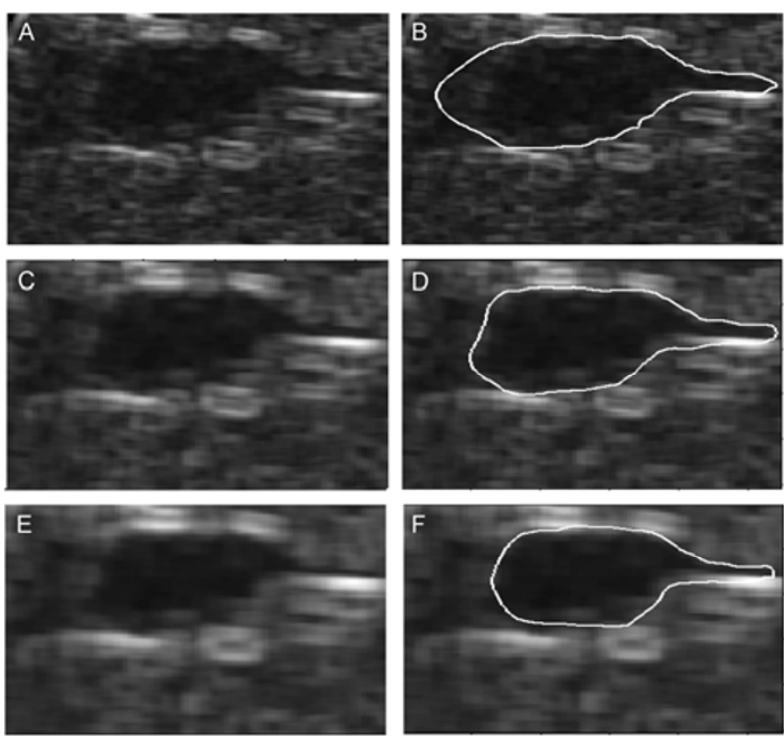

Figure 5 Image feature corresponding to the image presented in Figure $3 \mathrm{C}$. A, C and E, The image features $I_{\mathrm{SD}}$ for $3 \times 3,5 \times 5$ and $7 \times 7$ kernels extracted from the liver cyst ultrasound image. B, D and F, The active contours or snakes (in white) of boundaries derived using $3 \times 3,5 \times 5$ and $7 \times 7$ kernels.

tested using the following procedure. First, the physician manually drew the contours for each cyst as shown in Figure $3 \mathrm{~B}$ and $\mathrm{E}$. The ACM fitting ability for the original im- 
ages is presented in Figure $3 \mathrm{C}$ and $\mathrm{F}$. The images were then imported into Matlab and the automatic ACM was implemented. The image feature $I_{\mathrm{SD}}$ was generated using $3 \times 3$, $5 \times 5$ and $7 \times 7$ standard-deviation kernels. Figures 4 and $5 \mathrm{~A}$, $\mathrm{C}, \mathrm{E}$ illustrate the resulting image features. The ACM detection ability for image features is shown in Figures 4 and 5B, D, F.

Tables 1 and 2 give the effectiveness data for the proposed method in terms of the AER. Table 3 presents the same AER data to compare different methods for the same technique of segmentation. Finally, Table 4 gives the execution time for the ACM and watershed methods.

Table 3 AERs of breast and liver cyst ultrasound images segmented using the watershed method

\begin{tabular}{ccccc}
\hline & \multicolumn{4}{c}{ AER $(\%)$ in average } \\
\cline { 2 - 5 } Images & $\begin{array}{c}\text { Original } \\
\text { image }\end{array}$ & $\begin{array}{c}I_{\mathrm{SD}} \\
3 \times 3 \text { kernel } \\
\text { size }\end{array}$ & $\begin{array}{c}I_{\mathrm{SD}} \\
5 \times 5 \text { kernel } \\
\text { size }\end{array}$ & $\begin{array}{c}I_{\mathrm{SD}} \\
7 \times 7 \text { kernel } \\
\text { size }\end{array}$ \\
\hline $\begin{array}{c}\text { Synthetic images } \\
\text { Breast cyst images- } \\
\text { Average values }\end{array}$ & 3.25 & 2.96 & 1.62 & 1.78 \\
$\begin{array}{c}\text { Liver cyst images- } \\
\text { Average values }\end{array}$ & 31.17 & 25.64 & 21.26 & 20.41 \\
\hline
\end{tabular}

Table 4 Average of the convergence speeds for methods used (26 ultrasound images were tested)

\begin{tabular}{cccc}
\hline & \multicolumn{3}{c}{ Execution time $(\mathrm{s})$} \\
\cline { 2 - 4 } Segmentation method & $I_{\mathrm{SD}}$ & $I_{\mathrm{SD}}$ & $I_{\mathrm{SD}}$ \\
& $3 \times 3$ kernel & $5 \times 5$ kernel & $7 \times 7$ kernel \\
& size & size & size \\
\hline Active contour model & 2.09 & 1.45 & 1.01 \\
Watershed & 0.55 & 0.52 & 0.36 \\
\hline
\end{tabular}

The resolution variation of the image feature was quantitatively evaluated using the CG method. The CG values for the synthetic image and various masks were 41.11 for the original image, 62.05 for $I_{\mathrm{SD} 3 \times 3}, 72.36$ for $I_{\mathrm{SD} 5 \times 5}$, and 75.28 for $I_{\mathrm{SD} 7 \times 7}$. The average values of $\mathrm{CG}$ for the breast ultrasound images and masks were 46.8 for the original image, 69.78 for $I_{\mathrm{SD} 3 \times 3}, 77.98$ for $I_{\mathrm{SD} 5 \times 5}$, and 62.39 for $I_{\mathrm{SD} 7 \times 7}$. The average values of $\mathrm{CG}$ for ultrasound liver images were 54.76 for the original image, 78.54 for $I_{\mathrm{SD} 3 \times 3}, 87.02$ for $I_{\mathrm{SD} 5 \times 5}$, and 58.17 for $I_{\mathrm{SD} 7 \times 7}$.

\section{Discussion}

The textures of cysts in the breast and liver differ and cysts have varying degrees of visibility; thus cysts are difficult to segment. From a clinical point of view, cysts are generally benign, so there is no immediate clinical application of the segmentation; the results are meant to illustrate the operation of the first-order statistical feature and ACM techniques.
In the case of the new synthetic-image feature, obtained using a $5 \times 5$ kernel and presented in Figure $2 \mathrm{C}$, it is seen that the snake surrounds the disk very well and the best value of the AER ( $4.79 \%$ error relative to the real cyst area) was obtained. Figure 2B and D shows a local surpassing of the contour. The better edges, extracted using $I_{\mathrm{SD}}$ and the $5 \times 5$ kernel, resulted in much better final segmentation. Generally, as seen in Figures 4 and 5, regions were precisely segmented. It is worth mentioning that for in vivo images, the contours of the cyst were delineated very well when the $I_{\mathrm{SD}}$ image feature for the $5 \times 5 \mathrm{kernel}$ was used. The AER average value was $8.88 \%$ for the breast cyst and $10.82 \%$ for the liver cyst, and the accuracy of the ACM method was, on average, $91 \%$. Differences along the boundary were likely due to noise and speckle in the images.

To drive ACMs towards object boundaries, salient image features and strong edges are required. When the external energy function is minimized, the snake is attracted towards the edge points of the image and the shape of the snake is smoothed. We optimized the weighting parameters of the internal energy taking into account three issues: (i) when a snake having low elasticity and rigidity parameter values moves towards the object, it meets erroneous edge segments in the image but, finally, it finds a local minimum; (ii) a snake having mid-range parameter values surpasses the erroneous edge and moves towards the object and aligns itself closely around the contour of the object; (iii) a snake having highest parameter values shrinks and surpasses the contour of the object. The third case of surpassing the contour of the object is unacceptable and is related to the degeneration of the snake. Our choice was $\alpha=0.4$ for the elasticity parameter and $\beta=0.2$ for the rigidity parameter. These values were used for all snakes in all tested images (both synthetic and in vivo images).

The proposed method represents an improved approach because it takes into consideration the textures in local regions of the images rather than the texture analysis of the whole image. The immediate advantage is that we can analyze complex images. Additionally, this method combines global echogenicity information with local edge information. Considerable efforts were made to optimize this algorithm. It is anticipated that the optimization of the active contour segmentation algorithm depends on focal points (regions of interest determined manually), the distribution of pixel values due to speckle (kernel size), and choice of the energy function. If we choose an improper initial condition, the process provides a poor result or it requires a long processing time. The obtained results show that a large $7 \times 7$ kernel produces an $I_{\mathrm{SD}}$ image feature without strong edges and this model cannot correctly detect objects and it will tend to collapse. However, if the kernel is small (e.g., $3 \times 3$ ), then more iteration will be needed to scan the object. In our study, $I_{\mathrm{SD}}$ has strong edges for kernel dimensions of $5 \times 5$. However, the proposed method cannot replace the physician's practical experience. 
The limitation of the proposed algorithm is the manual interaction in fixing the contour and establishing the necessary number of iterations. For the synthetic image, 250 iterations were needed. For in vivo images, the number of iterations required for convergence was between 30 and 60. According to the results presented by Cvancarova et al. [9], liver tumor boundaries have been determined using about 40 iterations. Another drawback is the spatial positioning of the snake initialization. If the snake is initialized "too far" from the cyst boundary in terms of shape and position, it is possible that the contour may not converge onto the cyst boundary. The key observation here is that the snake algorithm says nothing about the initial location of a snake, and this must be manually determined by the physician. The active contour algorithm based on the image feature avoids the collapse of the snake when there is poor convergence in noisy images. As a final remark, we note that the parameters of the ACM can be better tuned for any ultrasound image regardless of the quality of the image.

Our primary objective in this work was to investigate the clinical utility of the image features extracted from ultrasound images and to detect the contours of breast and liver cysts using the ACM. The AER was used as an established tool for validating the segmentation accuracy. Our results are in accordance with the studies of Ciecholewski [10], who tested the ACM on ultrasound images to extract the shape of the gallbladder, and studies carried out by Seo et al. [31] on the automatic segmentation of the liver using computed tomography images. In both papers, the AER is used as an evaluation criterion of the performances.

From the experimental data presented in Tables 1 and 2, we conclude that the semi-automatic detection of contours of breast cysts using the ACM has better performance than the use of the original cyst breast ultrasound image when the $5 \times 5$ kernel is used. In the case of breast images, the AER is $14.90 \%$ for original images versus an average value of $8.88 \%$ for the $5 \times 5$ kernel. In the case of liver images, the result is $16.55 \%$ for the original image delineation versus $10.82 \%$ for the $5 \times 5$ kernel. The same result is obtained for the synthetic image $(5.64 \%$ for the original image versus $4.79 \%$ for the $5 \times 5$ kernel).

To qualify our contribution, we compared the proposed approach with the classical approach of the watershed method. Experimental results show that our method gives better results. Table 3 compares the AER results for the watershed method and our proposed method. The AER average value for the $5 \times 5 \mathrm{kernel}$ is $8.88 \%$ for the breast cyst and $10.82 \%$ for the liver cyst when the ACM is used, whereas the AER average value is $21.26 \%$ for breast images and $30.62 \%$ for liver images when the watershed method is used. Table 4 gives the execution times for the watershed and ACM methods. The time for the watershed method is considerably less than that for the ACM method, and consequently, the watershed algorithm is much faster in image segmentation. However, when we compare the AER values provided by Tables $1-3$, the segmentation results are more precise for the ACM while the watershed algorithm produces large errors.

Calculated CG values confirmed, as expected, that the $3 \times 3$ and $5 \times 5$ masks were better choices for fitting noisy images and were apt to lead towards better resolution for ultrasound images.

These results confirm the efficiency and utility of the image feature of the standard deviation of the gray-level values in the ACM. We also note that the size of the kernel is a critical factor in the successful application of this type of segmentation.

\section{Conclusion}

Segmentation remains a necessary stage in ultrasound imaging to obtain qualitative measurements such as the correct detection of the border of the object of interest. This paper presents a new approach to extract the shapes of breast and liver cysts from ultrasound images using image features developed with the standard deviation and ACM. In the case of contour detection using the image feature and a $5 \times 5$ kernel, the method correctly detected boundaries. As AER values were small, we conclude that replacing the image function with the standard deviation image feature provides good results in border detection.

We also compared our contribution with the watershed method. Although the ACM required approximately three times the computing time, the watershed segmentation method did not demonstrate explicit advantages over the proposed method.

This work was supported by the Project SOP HRD-EFICIENT 61445/2009 of University Dunarea de Jos of Galati, Romania.

1 Jahne B. Digital Image Processing. New York: Springer, 2002

2 Saini K, Dewal M L, Rohit M. Ultrasound imaging and image segmentation in the area of ultrasound: a review. Sci Tech, 2010, 24: 41-60

3 Noble J A, Boukerroui D. Ultrasound image segmentation: a survey. IEEE Trans Med Imaging, 2006, 25: 987-1010

4 Vard A R, Monadjemi A H, Jamshidi K, et al. Fast texture energy based image segmentation using Directional Walsh-Hadamard Transform and parametric active contour models. Expert Syst Appl, 2011, 38: 11722-11729

5 Kass M, Witkin A, Terzopoulos D. Snakes: Active contour models. Int J Comput Vision, 1988, 1: 321-331

6 Terzopolus D. The computation of visible-surface representation. IEEE T Pattern Anal, 1988, 10: 17-22

7 Lefebvre F, Berger G, Laugier P. Automatic detection of the boundary of the calcaneus from ultrasound parametric images using an active contour model. IEEE Trans Med Imaging, 1998, 17: 45-52

8 Maroulis D E, Savelonas M A, Iakovidis D K, et al. Variable background active contour model for computer-aided delineation of nodules in thyroid ultrasound images. IEEE Trans Inf Technol B, 2007, 11: 537-544

9 Cvancarova M, Albregtsen F, Brabrand K, et al. Segmentation of ultrasound images of liver tumors applying snake algorithms and GVF 
computer. In: Proceeding of International Congress Series on Assisted Radiology and Surgery, Berlin, Germany, 2005. 218-223

10 Ciecholewski M. Gallbladder boundary segmentation from ultrasound images using active contour model. Lect Notes Comput Sc, 2010, 6283: 63-69

11 Cheng D, Trucksass A, Cheng K, et al. Automatic detection of the intimal and the adventitial layers of the common carotid artery wall in ultrasound B-mode images using snakes. In: Proceeding of International Conference on Image Analysis and Processing, Venice, Italy, 1999. 452-457

12 Mao F, Gill J, Downey D A, et al. Segmentation of carotid artery in ultrasound images: method development and evaluation technique. Med Phys, 2000, 27: 1961-1970

13 Loizou C, Pattichis C. Snakes based segmentation of the common carotid artery intima media. Med Biol Comput, 2007, 45: 35-49

14 Chen D R, Chang R F, Wu W J, et al. 3-D breast ultrasound segmentation using active contour model. Ultrasound Med Biol, 2003, 29: 1017-1026

15 Huang Y L, Chen D R. Automatic contouring for breast tumors in 2-D sonography. IEEE Eng Med Biol Soc, 2005, 3: 3225-3228

16 Evans A, Nixon M. Biased motion-adaptive temporal filtering for speckle reduction in echocardiography. IEEE Trans Med Imaging, 1996, 15: 39-50

17 Chalana V L, Haynor D T, Yongmin Kim D R, et al. A multiple active contour model for cardiac boundary detection on echocardiographic sequences. IEEE Trans Med Imaging, 1996, 15: 290-298

18 Mudabhushi A, Metaxas D N. Combining low-, high-level and empirical domain knowledge for automated segmentation of ultrasonic breast lesions. IEEE Trans Med Imaging, 2003, 22: 155-169

19 Su Y, Wang Y, Jiao Y, et al. Automatic detection and classification of breast tumors in ultrasonic images using texture and morphological features. Open Med Inform J, 2011, 5: 26-37
20 Kim P, Lee Y, Jung Y, et al. Liver extraction in the abdominal CT image by watershed segmentation algorithm, In: Proceedings of World Congress on Medical Physics and Biomedical Engineering, Seoul, Korea, 2006. 2563-2566

21 Huang Y L, Chen D R. Watershed segmentation for breast tumor in 2-D sonography. Ultrasound Med Biol, 2004, 30: 625-632

22 Sharma A, Kohli P G, Kapoor D S, et al. Qualitative analysis of membrane filter used for bacteria filtration using feature extraction techniques. IJCEM, 2011, 13: 70-77

23 Zhao C G, Zhuang T G. A hybrid boundary detection algorithm based on watershed and snake. Pattern Recogn Lett, 2005, 26: 1256-1265

24 Ishitani T, Sato M. Contrast-to-gradient method for the evaluation of image resolution in scanning electron microscopy. J Electron Microsc, 2002, 51: 369-382

25 Xu C, Prince J L. Snakes, shapes, and gradient vector flow. IEEE Trans Image Process, 1998, 7: 359-369

26 Almageed W A, Smith C E. Active deformable models using density estimation. Int J Image Graph, 2004 4: 343-361

27 Vard A R, Nilchi N, Moallem P. Object detection and image segmentation using texture pressure energy in parametric active contour models. J Chin Inst of Eng, 2008, 31: 649-657

28 Ivins J, Porrill J. Active region models for segmenting medical images. In: Proceedings of IEEE International Conference on Image Processing, Austin, USA, 1994. 227-231

29 Nurhayati O D, Widodo T S, Susanto A, et al. First order statistical feature for breast cancer detection using thermal images. World Acad Sci, 2010, 70: 1040-1073

30 Schulze P. Macromedia Fireworks 8: Training from the Source. San Francisco: Macromedia Press, 2005

31 Seo K S, Kim H B, Park T, et al. Automatic liver segmentation of contrast enhanced CT images based on histogram processing. Comput Inf Sci, 2005, 3610: 1027-1030

Open Access This article is distributed under the terms of the Creative Commons Attribution License which permits any use, distribution, and reproduction in any medium, provided the original author(s) and source are credited. 